\title{
Effects of Hypoplastic Fetal Umbilical Artery on Doppler Findings For Common Iliac Arteries
}

\author{
Asli Tanrivermis Sayit, ${ }^{1,}$ Ali Ipek, ${ }^{2}$ Ilkay Sedakat Idilman, ${ }^{2}$ Nurdan Cay, ${ }^{2}$ Pinar Gunbey, ${ }^{3}$ and Mustafa \\ Karaoglanoglu ${ }^{2}$ \\ ${ }^{1}$ Department of Radiology, Samsun Gazi State Hospital, Samsun, Turkey \\ ${ }^{2}$ Department of Radiology, Ankara Ataturk Education and Research Hospital, Ankara, Turkey \\ ${ }^{3}$ Department of Radiology, Ondokuz Mayis University, Samsun, Turkey \\ "Corresponding author: Asli Tanrivermis Sayit, Department of Radiology, Samsun Gazi State Hospital, Samsun, Turkey. Tel: +90-5324949082, Fax: +90-3624576091, E-mail: \\ draslitanrivermissayit@gmail.com
}

Received 2014 September 13; Revised 2015 February 17; Accepted 2015 April 02.

\begin{abstract}
Background: The umbilical cord contains two arteries and one vein. Normally, the diameters of umbilical arteries are close in size, but this may vary, as one umbilical artery may be considerably smaller or more hypoplastic than the other.

Objectives: This study aimed to investigate the effect of hypoplastic umbilical arteries on Doppler ultrasonography (US) findings for the common iliac arteries (CIAs), such as the pulsatility index (PI) and resistive index (RI) values, and to investigate the associations between hypoplastic fetal umbilical arteries and placental, umbilical cord, and fetal pathologies.

Patients and Methods: Doppler US was performed on 165 pregnant women between 18 and 22 weeks of gestation, and again between 27 and 37 weeks of gestation. The umbilical artery diameters and both CIA flow parameters (PI and RI) were measured during these visits. The presence of a diameter difference of greater than $25 \%$ (95th percentile) between the two umbilical arteries was accepted as a hypoplastic umbilical artery.

Results: Eleven of the 165 patients (6.7\%) demonstrated a hypoplastic umbilical artery. Second-trimester US showed median dominant and non-dominant CIA PI values of 1.43 and $1.60(P=0.062)$, respectively, and median dominant and non-dominant CIA RI values of 0.80 and $0.85(\mathrm{P}<0.001)$, respectively, in the hypoplastic umbilical artery group. Third-trimester US showed median dominant and non-dominant CIA PI values of 1.24 and $1.66(\mathrm{P}=0.062)$, respectively, and median dominant and non-dominant CIA RI values of 0.73 and $0.82(\mathrm{P}=0.002)$, respectively, in the hypoplastic umbilical artery group. We did not observe an association between hypoplastic umbilical arteries and placental, umbilical cord, or fetal pathologies.

Conclusion: Our study revealed that the hypoplastic umbilical artery group's non-dominant CIA had higher RI values than the contralateral dominant CIA.
\end{abstract}

Keywords: Umbilical Arteries, Doppler Ultrasonography, Iliac Arteries

\section{Background}

The umbilical cord contains one vein and two arteries, which transport viable materials to the fetus and metabolites to the placenta. In general, similarly sized umbilical arteries are expected. However, differently sized umbilical arteries are detected in approximately $0.7 \%-1.4 \%$ of pregnancies $(1,2)$. Some of these fetuses have a hypoplastic umbilical artery, which is defined as a significantly smaller umbilical artery in comparison with the contralateral one. The level of significance differs according to various authors (2-4). Some define it as a difference of $>2 \mathrm{~mm}$ (3), whereas others suggest a difference of $50 \%$ or more compared to the dominant artery (4). In a previous study, differently sized umbilical arteries were shown to cause significant changes in blood-flow parameters (2). Studies investigating the impact of discordant umbilical arteries on clin- ical outcomes have demonstrated conflicting results. In some studies, a hypoplastic umbilical artery was found to be associated with umbilical cord hematoma, placental infarction, trisomy 18, polyhydramnios, congenital heart disease, intrauterine growth retardation (IUGR), and abnormal insertion of the umbilical cord (4-7). However, in another study, no association between umbilical or placental abnormalities and discordant umbilical arteries was found (2).

In fetuses with a single umbilical artery (SUA), all of the fetal blood travels to the placenta via the vessel on the side of the umbilical artery. On the contralateral side, where the umbilical artery is absent, the common iliac artery (CIA) cannot participate in fetoplacental circulation and carries the blood to the lower limbs. Studies investigating the relationship between SUA and differences in the diameter of the CIA and related Doppler ultrasonography (US) param- 
eters are available in the literature $(1,8)$. Part of the conclusions of these studies is that the CIA is hypoplastic on the side on which the umbilical artery is absent. Raio et al. also found higher resistive index (RI) values in hypoplastic umbilical arteries as compared with the contralateral side (7). However, studies investigating the flow parameters between a hypoplastic umbilical artery and the ipsilateral CIA are not available in the literature.

\section{Objectives}

In the present study, we aimed to investigate the effects of hypoplastic umbilical arteries on Doppler findings for the CIAs, and to investigate the association between hypoplastic fetal umbilical arteries and placental, umbilical cord, and fetal pathologies.

\section{Patients and Methods}

This research study was approved by our institutional human ethics committee. All patients were informed about the procedures, and their approval was obtained. Routine obstetric US during the $18^{\text {th }}$ to $22^{\text {nd }}$ gestational weeks was performed on 212 pregnant women who were admitted to our clinic for evaluation for congenital fetal anomalies. Single pregnancies that had 1, a known date of the last menstrual period; 2, both umbilical arteries; 3, intact membranes; 4, 3-vessel cords; 5, prenatal followup; and 6, postnatal medical records were included in this study. Multiple pregnancies and fetuses with inadequately imaged umbilical cords were excluded.

All ultrasound examinations were performed with a GE Logic 9 (Milwaukee, USA) ultrasound machine using a 3.5-5 MHz multi-frequency convex probe operated by a specialist experienced in obstetric US (A.I., 18 years of experience). Detailed obstetric US was performed on all fetuses, and minor anomalies were noted. Fetal abdominal circumference, femur length, and biparietal diameter were measured, and the average was used to confirm gestational age.

The umbilical cord structure and the presence of two arteries and one vein were carefully evaluated. An umbilical artery measurement was taken from the free section of the cord away from the fetus and the placenta, and the diameter was measured from the inner to the outer lumen of the artery (Figure 1A-1B). The percentage difference in diameter between the umbilical arteries (\%AA) was calculated using the following formula (2):

$\% A A=\frac{A 1-A 2}{A 1}$

In this formula, A1 and A2 represent the largest and smallest diameters of the umbilical arteries, respectively.
According to this formula, two groups were created. Group A (the hypoplastic umbilical artery group) consisted of those with a $>25 \%$ ( $>95^{\text {th }}$ percentile) difference in umbilical artery diameters, and group B (the control group) consisted of those with $\mathrm{a}<25 \%$ ( $<95^{\text {th }}$ percentile) difference in the umbilical artery diameters.

CIA diameters were assessed via magnified grayscale images, and the larger of these was accepted as dominant (7). Accordingly, both CIAs were examined just distal to the aortic bifurcation via color Doppler US, and the pulsatility index (PI) and RI values were measured (Figure 1C). Followup US examinations were performed between gestational weeks 27 and 37, and the measurement of these parameters was repeated. The insonation angle between the umbilical cord vessels and the Doppler waves was between $30^{\circ}$ and $60^{\circ}$ in all Doppler studies. The pulse Doppler gain was -10 to $10 \mathrm{~dB}$, the pulse gate was $2-4 \mathrm{~mm}$, and a minimal wall filter was used.

Maternal and pregnancy characteristics; placental, umbilical cord, and fetal pathologies; amniotic fluid quantity; meconium staining; APGAR score; birth weight; and the birth route of the infant were compared among the groups and subgroups.

Statistical analysis was performed using SPSS (version 15.0, SPSS Inc., Chicago, IL, USA) computer software. The Bonferroni-corrected Wilcoxon signed-rank test was used for comparing the intra-group medians for the left and right measurements when the distribution was not normal. The Mann-Whitney U test was used for two-group comparisons, and the Kruskal-Wallis test was used for three-group comparisons of inter-group medians when a normal distribution was absent. The Chi-square test was used to compare percentages between groups, and Student's t-test was used for comparing the dominant and non-dominant CIA RI values when the distribution was normal. A P value of $<0.05$ was considered significant in all statistical analyses. All data were expressed as median $\pm \mathrm{SD}$.

\section{Results}

Obstetric US examinations were performed on 212 pregnant women between their 18th and 22nd weeks of gestation (early second-trimester US). A single umbilical artery was detected in five women. Forty-two patients were excluded because their follow-up US examinations were missing. Therefore, 47 cases were excluded from the study, and a total of 165 patients were included. Of these, 11 demonstrated a hypoplastic umbilical artery, indicating an incidence of $6.7 \%$. The hypoplastic umbilical arteries were found to be predominantly on the left side (8/11). 

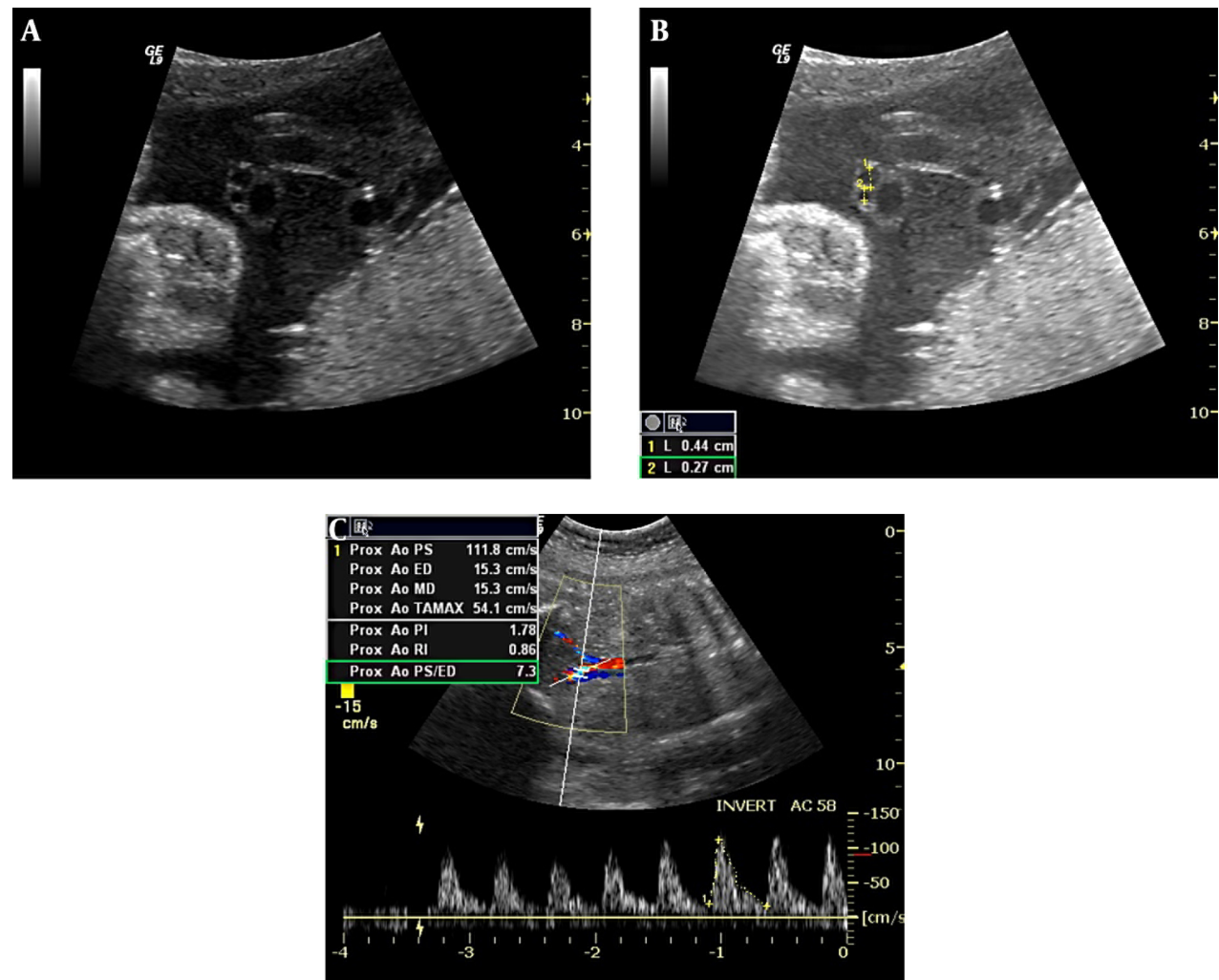

Figure 1. Transverse view of an umbilical cord showing the size difference between umbilical arteries (A). The measurement of the umbilical arteries reveals the difference in a fetus at 30 weeks, 6 days of gestation (B). Non-dominant CIA flow waveforms of the same fetus demonstrate a high RI value (C).

The mean patient age was 27 years in group A and 25 years in group B. Mean gravidity and parity were $2 \pm 0.94$ (median, 2; range, 1 - 4) and $1 \pm 0.5$ (median, 1 ; range, 0 1) for group A, respectively, and $2 \pm 1$ (median, 2; range, 1 6) and $1 \pm 0.77$ (median, 1 ; range, 0 - 4) for group B, respectively. There was no significant difference between the two groups in terms of maternal age, gravidity, or parity $(\mathrm{P}=$ $0.91,0.33$, and 0.82, respectively). Group A's third-trimester US confirmed the diagnosis of hypoplastic umbilical artery in all 11 of the subjects.

The median diameters of the large and small umbilical arteries of group A upon second-trimester US were 2 $\pm 0.28 \mathrm{~mm}$ and $1.2 \pm 0.24 \mathrm{~mm}(\mathrm{P}<0.001)$, respectively. The median diameters of the large and small umbilical arteries of group A upon third-trimester US were $3.4 \pm 0.53$ $\mathrm{mm}$ and $2.7 \pm 0.61 \mathrm{~mm}(\mathrm{P}=0.001)$, respectively. The median diameters of the large and small umbilical arteries of group B upon second-trimester US were $1.8 \pm 0.27 \mathrm{~mm}$ and $1.7 \pm 0.27 \mathrm{~mm}$, respectively. The median diameters of the large and small umbilical arteries of group B upon thirdtrimester US were $3.2 \pm 0.47 \mathrm{~mm}$ and $3.0 \pm 0.47 \mathrm{~mm}$, respectively. When we compared the median RI and PI val- ues of the dominant and non-dominant CIAs, we observed a significant difference between the dominant and nondominant CIA RI values of group $A$ in the second and third trimesters $(\mathrm{P}<0.001$ and $\mathrm{P}=0.002$, respectively). We also observed a significant difference between the dominant and non-dominant CIA PI values of group A in the third trimester $(\mathrm{P}=0.005)$. We did not observe a significant difference between the dominant and non-dominant CIA RI and PI values of group B in the second and third trimesters. The Doppler characteristics of the CIAs are summarized in Table 1.

The birth weights of the fetuses in group A and group B were $3060 \pm 418$ grams and $3185 \pm 521$ grams, respectively, with no significant difference $(\mathrm{P}=0.90)$. There was no statistically significant difference between the two groups in terms of umbilical cord pathology, nuchal cord, 5-minute APGAR score, and the nature of the amniotic fluid ( $\mathrm{P}>$ 0.05).

Nine of 11 patients in group A delivered at term via spontaneous vaginal delivery, and the other two patients delivered via cesarean section. There were no patients with hypertension, preterm labor, or premature rupture of 
Table 1. Doppler Characteristics of CIAs According to Groups and Trimesters

\begin{tabular}{|c|c|c|c|c|c|}
\hline & & & Dominant CIA & Non-Dominant CIA & PValue \\
\hline \multirow{4}{*}{ Group A: (hypoplastic umbilical artery) } & \multirow{2}{*}{ Second trimester } & RI & $0.8(0.66-0.85)$ & $0.85(0.72-0.91)$ & $<0.001$ \\
\hline & & PI & $1.43(1.01-1.76)$ & $1.60(1.05-2.89)$ & 0.062 \\
\hline & \multirow{2}{*}{ Third trimester } & $\mathrm{RI}$ & $0.73(0.63-0.86)$ & $0.82(0.68-0.9)$ & 0.002 \\
\hline & & PI & $1.24(1.10-1.85)$ & $1.66(1.13-2.15)$ & 0.005 \\
\hline \multirow{4}{*}{ Group B: (control) } & \multirow{2}{*}{ Second trimester } & $\mathrm{RI}$ & $0.83(0.66-0.93)$ & $0.83(0.64-0.96)$ & $>0.05$ \\
\hline & & PI & $1.59(0.90-2.80)$ & $1.58(0.94-3.02)$ & $>0.05$ \\
\hline & \multirow{2}{*}{ Third trimester } & $\mathrm{RI}$ & $0.76(0.60-0.94)$ & $0.78(0.56-0.93)$ & $>0.05$ \\
\hline & & PI & $1.35(0.24-2.18)$ & $1.40(0.77-2.41)$ & $>0.05$ \\
\hline
\end{tabular}

Abbreviations: CIA, common iliac artery; RI, resistive index; PI, pulsatility index.

membranes in group A.

In our study, no placental or umbilical cord pathology was detected in group A. The detected associated abnormalities among the group A patients were polyhydramnios in two cases, meconium-stained amniotic fluid in four cases, and nuchal cord in two cases. No chromosomal defects, fetal anomalies, or syndromes were detected among the group A patients. Finally, gestational diabetes was found in only one pregnant woman from group A.

\section{Discussion}

Hypoplastic umbilical artery is accepted as an incomplete form of SUA (4). Although its etiology is unknown, this condition has been found to be associated with various pathologies, such as trisomy 18, polyhydramnios, congenital heart disease, IUGR, placental infarction, umbilical cord hematoma, and abnormal umbilical cord insertion (5-7). Dolkart et al. studied umbilical artery diameters and found a $2 \mathrm{~mm}$ difference in six cases measured in the second and third trimesters. One of these six cases was accompanied by preterm birth, and another resulted in death due to neonatal liver failure three months after birth. No clinical pathology was reported in the other four cases (9). In another study by Predanic et al., no pathological findings were detected during the examination of the placental and umbilical cords of 12 pregnancies demonstrating significant discordance $(>29.5 \%$, or the 95th percentile) between the two umbilical artery diameters (2). Conversely, Raio et al. demonstrated abnormal cord insertions (marginal, velamentous, and eccentric) in eight of 14 cases with $>1 \mathrm{~mm}$ differences between the umbilical artery diameters. Placental abnormalities were detected in the remaining seven cases (three placental infarctions, two bipartite placentas, one placenta succenturiata, one chorangioma, and one absence of Hyrtl anastomosis) (7).
Petrikovsky and Schneider detected hypoplastic umbilical arteries using prenatal US in 12 patients. They found one of these cases to be associated with trisomy 18, three with polyhydramnios, one with congenital heart disease, and two with IUGR. They also found maternal diabetes in four cases (4). In our study, no placental, umbilical cord, or fetal pathologies were detected in the patients with hypoplastic umbilical arteries. However, two pregnancies were complicated by polyhydramnios, four by meconium staining, and one by gestational diabetes.

Sepulveda et al. examined blood-flow patterns in the CIA in 15 cases of SUA. They found that PI values on the side not attending to fetoplacental circulation were higher than on the contralateral side (8). In our study, the hypoplastic umbilical artery group demonstrated higher PI and RI values for the non-dominant CIAs in comparison with the dominant side during the second trimester. The higher RI values may have been due to the smaller diameter of the CIA on the side of the hypoplastic umbilical artery at early gestational stages.

In a study of eight cases of SUA from birth to 4 years of age, Meyer et al. reported significant anatomical and histological differences between the CIAs. Whereas a normal muscular structure was detected in the smaller CIA, a calcified pattern was detected in the larger CIA. Also, interestingly, early atherosclerotic lesions were identified in the larger vessel. It has been thought that this may reduce blood flow, especially in the dominant vessel, resulting in vessel-wall remodeling after the clamping of the cord (10).

The small sample size and the lack of any macroscopic examinations of the placental and umbilical cord pathologies are some of the limitations of our study. The effect of hypoplastic fetal umbilical artery on the prognosis is difficult to judge definitively due to the small sample size. However, it is known that the fetal prognosis is better for hypoplastic umbilical arteries compared with SUAs. The inad- 
equate number of fetuses with hypoplastic umbilical arteries is a limitation of our study. More extensive studies are needed to determine the effects of hypoplastic fetal umbilical artery on the fetal prognosis. The present study can act as a pioneer for future studies with larger sample sizes. Another limitation of this study was the subjective evaluation criteria used for the dominant and non-dominant fetal CIAs. However, there is no superior technique available for the evaluation of small fetal structures, such as the umbilical and iliac arteries.

In conclusion, our study revealed that the hypoplastic umbilical artery group's non-dominant CIA had higher RI values in comparison with the contralateral dominant CIA. We did not observe an association between hypoplastic umbilical arteries and placental, umbilical cord, or fetal pathologies.

\section{Acknowledgments}

There is no acknowledgements.

\section{Footnotes}

Authors' Contribution: Asli Tanrivermis Sayit, Ali Ipek, and Ilkay Sedakat Idilman diagnosed the patients and prepared the article. Nurdan Cay, Pinar Gunbey, and Mustafa Karaoglanoglu helped to prepare the article.

Financial Disclosure: There is no financial disclosure. Funding/Support: There is no funding and support

\section{References}

1. Weissman A, Drugan A. Sonographic findings of the umbilical cord: implications for the risk of fetal chromosomal anomalies. Ultrasound Obstet Gynecol. 2001;17(6):536-41. doi: 10.1046/j.14690705.2001.00408.x. [PubMed: 11422981].

2. Predanic M, Perni SC. Antenatal assessment of discordant umbilical arteries in singleton pregnancies. Croat Med J. 2006;47(5):701-8. [PubMed: 17042061].

3. Sepulveda W, Flack NJ, Bower S, Fisk NM. The value of color Doppler ultrasound in the prenatal diagnosis of hypoplastic umbilical artery. Ultrasound Obstet Gynecol. 1994;4(2):143-6. doi: 10.1046/j.14690705.1994.04020143.x. [PubMed: 12797209].

4. Petrikovsky B, Schneider E. Prenatal diagnosis and clinical significance of hypoplastic umbilical artery. Prenat Diagn. 1996;16(10):93840. doi: 10.1002/(SICI)1097-0223(199610)16:10<938::AIDPD964>3.0.CO;2-S. [PubMed: 8938065].

5. Sepulveda W, Shennan AH, Bower S, Fisk NM. Discordant umbilical artery flow velocity waveforms in spontaneous umbilical cord hematoma. J Clin Ultrasound. 1995;23(5):330-2. [PubMed: 7642775].

6. Harper MA, Murnaghan GA. Discordant umbilical artery flow velocity waveforms and pregnancy outcome. Br J Obstet Gynaecol. 1989;96(12):1449-50. [PubMed: 2695160].

7. Raio L, Ghezzi F, Di Naro E, Gomez R, Saile G, Bruhwiler H. The clinical significance of antenatal detection of discordant umbilical arteries. Obstet Gynecol. 1998;91(1):86-91. [PubMed: 9464727].

8. Sepulveda W, Nicolaidis P, Bower S, Ridout DA, Fisk NM. Common iliac artery flow velocity waveforms in fetuses with a single umbilical artery: a longitudinal study. Br J Obstet Gynaecol. 1996;103(7):660-3. [PubMed: 8688392].

9. Dolkart LA, Reimers FT, Kuonen CA. Discordant umbilical arteries: ultrasonographic and Doppler analysis. Obstet Gynecol. 1992;79(1):5963. [PubMed: 1727588].

10. Meyer WW, Lind J. Iliac arteries in children with a single umbilical artery. Structure, calcifications, and early atherosclerotic lesions. Arch Dis Child. 1974;49(9):671-9. [PubMed: 4418232]. 